\title{
The deoxyguanosine kinase gene is mutated in individuals with depleted hepatocerebral mitochondrial DNA
}

H. Mandel, R. Szargel, V. Labay, O. Elpeleg, A. Saada, A. Shalata, Y. Anbinder, D. Berkowitz, C. Hartman, M. Barak, S. Eriksson \& N. Cohen

Nature Genet. 29, 337-341 (2001).

The legend of Fig. 4 contains two incorrect lane references in the second sentence. A corrected legend follows.

Fig. 4 Western-blot analysis of liver homogenate. We separated an equivalent of $2 \mathrm{mU}$ citrate synthase from affected individual 21 of family 1-F (lane 2) and three normal control individuals (lanes 1, 3 and 4) using SDS-PAGE, electroblotted the synthase and incubated it with anti-dGK antibodies. Molecular weight markers were run in the flanking slots. The DGK band is near the MW 29,000 band. The presence of a number of bands weakly cross-reactive with the DGK polyclonal antiserum shows that the proteins in the affected individual's sample were not affected.

\section{Genome scanning with array CGH delineates regional alterations in mouse islet carcinomas}

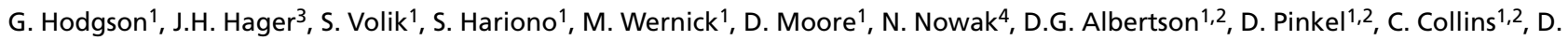
Hanahan $^{3}$ \& J.W. Gray ${ }^{1,2}$

Nature Genet. 29, 459-464 (2001).

Our paper was published online on 5 November. Regrettably, we inadvertantly omitted Norma Nowak from the list of authors. The correct list appears above; the corrected list of affiliations follows.

${ }^{1}$ Cancer Genetics and Breast Oncology Programs, UCSF Cancer Center, University of California at San Francisco, Box 0808, San Francisco, California 94143-0808, USA. ${ }^{2}$ Department of Laboratory Medicine, University of California at San Francisco, Box 0808, San Francisco, California 94143-0808, USA. ${ }^{3}$ Department of Biochemistry and Biophysics, UCSF Diabetes and Comprehensive Cancer Centers, University of California at San Francisco, San Francisco, California 94143-0534, USA. ${ }^{4}$ Department of Human Genetics, Roswell Park Cancer Institute, Buffalo, New York 14263, USA.

\section{Mutations in the protein tyrosine kinase gene, PTPN11, cause Noonan syndrome}

M. Tartaglia, E.L. Mehler, R. Goldberg, G. Zampino, H.G. Brunner, H. Kremer, I. van der Burgt, A.H. Crosby, A. Ion, S. Jeffery, K. Kalidas, M.A. Patton, R.S. Kucherlapati \& B. Gelb

Nature Genet. 29, 465-468 (2001).

Regrettably, we did not provide acknowledgment of two sources of funding before our paper was published online on 12 November. Full acknowledgment follows.

\section{Acknowledgments}

We thank the individuals with Noonan syndrome and their families who participated in this study, the physicians who referred the subjects, X. Song for technical assistance, $H$. Weinstein for insightful suggestions about the structural analysis, S. Hassan for providing algorithms and programs to carry out the Montre Carlo calculations and for discussions and G. Diaz and Y. Ioannou for reading the manuscript. This work was supported in part by grants from the NIH (to B.D.G., E.L.M. and R.K.) and from the Human Genetics Program at the Albert Einstein College of Medicine (to R.K.). We would like to thank the Birth Defects Foundation (UK) and the British Heart Foundation (PG /98191) for their support of this project. 